\title{
High-Fidelity Charring Ablator Thermal Response Model
}

\author{
N. N. Mansour* J. R. Lachaud ${ }^{\dagger} \quad$ T. E. Magin ${ }^{\ddagger} \quad$ J. de Muelenaere ${ }^{\S}$ \\ Y.-K Chen 9 \\ NASA Ames Research Center, Moffett Field, CA, 94035, USA
}

\section{Introduction}

Low-density carbon/phenolic is a class of ablative materials that is attractive for space exploration missions that use blunt bodies where weight and performance of the material are of primary importance, but shape preservation is not critical. Phenolic Impregnated Ceramic Ablator (PICA) is a material of this family develop by NASA for high-speed atmospheric entry mission. ${ }^{1}$ PICA has gained heritage with the success of the Stardust mission. ${ }^{2}$ Its performance has been extensively tested in support of the Constellation program and the Mars Science Laboratory (MSL) aeroshell design. PICA-X, also of the same class, will be used on Dragon, the commercial Space-X capsule ${ }^{\mathrm{a}}$. The European Space Agency (ESA) is currently supporting the development of a light weight Carbon-Resin ablator that could be used for sample return missions. ${ }^{3}$ Important for the scientific community, the MSL aeroshell has been instrumented, and extensive flight data for this material is expected in 2011 from the MEDLI project.

Current material response models were inspired from the model of Kendall et al. ${ }^{4}$ published in 1968. They are based on five major assumptions: 1) the elemental composition of the pyrolysis gases is not a function of the decomposition phases; 2) pyrolysis gases are transported by convection only; 3) air does not penetrate inside the material; 4) ablation only occurs at the surface; 5) the solid at the surface is in chemical equilibrium with the gas. In other words, from the extremely complex phenomena occurring in a porous ablative material, only Fourier's heat transfer and the pyrolysis of the solid are modeled. Interestingly, this simplified model has been able to reproduce within a reasonable accuracy, Arc Jet performance tests carried out on PICA in conditions relevant to NASA's missions. Therefore, depending on the design layout and quantity of interest, current models are robust. In off design conditions, however, there is a strong need to improve current models.

Weaknesses of current models become evident when specific objectives are desired. For instance, current studies by Gnoffo et al. ${ }^{5}$ have shown that at high atmospheric entry speeds, coupling the material response to the fluid code has an important effect on our ability to determine the flow environment that a mission will encounter. This study clearly points to the importance of knowing the composition of the gases being injected into the boundary layer. Another well known weakness of current models is that they do not take into account the effects of the micro-structure of the material on the performance of the material.

A new effort has been initiated to develop a high-fidelity Charring Ablator Thermal response model (CAT) with the goals to implement phenomenological models for 1) the decomposition chemistry of the matrix, 2) the flow within and chemical interactions of the pyrolysis gases with the porous medium and 3) the microstrucutre evolution of the material as it decomposes and ablates. In the paper we will present the implementation of a capability to keep track of the decomposition chemistry and the flow through the porous media. We evaluate the impact of the pyrolysis chemistry on the temperature response.

\footnotetext{
${ }^{*}$ Chief Division Scientist for Modeling and Simulation, Entry Systems and Technologies Division, Mail Stop 229-3, and AIAA Associate fellow.

${ }^{\dagger}$ Research Scientist, UARC, UC Santa Cruz, NASA Ames Research Center, Mail Stop 230-3.

¥Assistant Professor, Von Karman Institute for Fluid Mechanics, Brussels, Belgium

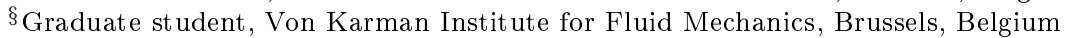

ฯAerospace Engineer, Aerothermodynamics Branch, Mail Stop 230-3

${ }^{a}$ http://www.spacex.com/press.php?page $=20090223$
} 


\section{Governing equations}

The governing equations describing the material response are the conservation of mass, species, momentum and energy in a porous medium. We will not formally derive the equations from first principles, but we point out that all dependent variables are assumed to be volume-averaged,

$$
\epsilon_{v}\langle\bullet\rangle=\frac{1}{V} \int_{V} \bullet d V
$$

where $V$ is a volume large compared to the scale of the fibers, but small compared to the scale of interest. $\epsilon_{v}$ is the porosity defined to be the ratio of the volume where the variable, $\bullet$, is non-zero in the averaging volume divided by the averaging volume.

For PICA, we shall keep track of the three quantities that constitute our composite material. These are the fibers, the matrix and the gas phase. We define three porosities, $\epsilon_{g}$, the gas phase volume fraction, $\epsilon_{f}$, the volume fraction occupied by the fibers, and $\epsilon_{m}$, the volume fraction occupied by the matrix. We shall also drop the averaging symbol for simplicity of notation.

\section{II.A. Gaseous species conservation}

The gas species are generated by the decomposition of the matrix and reactions among the species. The conservation equations read,

$$
\frac{\partial}{\partial t}\left(\epsilon_{g} X_{i}\right)+\frac{\partial}{\partial x}\left(\epsilon_{g} X_{i} v_{g}\right)=\pi_{i}+\epsilon_{g} \omega_{i}
$$

where the mole density of the gaseous species is

$$
X_{i}=\frac{p}{\mathcal{R} T} x_{i}
$$

and $x_{i}$ is the mole fraction of species $i$, with $i \in\left[1, N_{s}\right]$ where $N_{s}$ is the total number of active species. $p$, $T$, and $\mathcal{R}$ are the pressure, temperature and universal gas constant. $\pi_{i}$ is the production of species $i$ by the decomposition of the matrix, and $\omega_{i}$ is the sum of all sources/sinks from the reactions involving species $i$. While the mobility of the species due to diffusion plays a critical role in modeling material response, we have dropped the diffusion term for simplicity of the equation, and we have not included them in the results that we will be presenting.

\section{II.B. Pyrolysis}

We shall assume that the matrix decomposes in discrete stages, $j \in\left[1, N_{p}\right]$, where $N_{p}$ is the number of pyrolysis reactions

$$
P F R_{j} \rightarrow \sum_{i=1}^{N_{s}} \gamma_{j i} X_{i}
$$

$P F R_{j}$ is a fictive solid species of the phenolic formaldehyde resin (PFR), that produces $\gamma_{j i} X_{i}\left(i \in\left[1, N_{s}\right]\right)$ species as it decomposes. Note that $\gamma_{j i}$ is the molar composition of a mole of gas produced by the decomposition reaction, $j$, i.e. $\sum_{i=1}^{N_{s}} \gamma_{j i}=1$.

We define $F_{j}$ to be the fraction of the density loss due to decomposition reaction, $j$. The progress of the decomposition reaction, $P F R_{j}$, is tracked through a progress variable, $\xi_{j}$, that varies between 0 for the virgin material and 1 when all of the reactions are complete. The density of the matrix, $\rho_{m}$, in terms of these progress variables is then,

$$
\rho_{m}=\rho_{m v}-\delta \rho_{p} \sum_{j=1}^{N_{p}} F_{j} \xi_{j}
$$

where $\delta \rho_{p}=\left(\rho_{m v}-\rho_{m c}\right)$, in which $\rho_{m v}$ is the density of the virgin matrix, and $\rho_{m c}$ is the density of the charred matrix. By definition, $\sum_{j=1}^{N_{p}} F_{j}=1$, i.e. when $\xi_{j}=1$ for all $\mathrm{j}$. We assume that the rate of progress of the decomposition reaction is a function of the temperature only and is of the form, 


$$
\frac{\partial \xi_{j}}{\partial t}=\left(1-\xi_{j}\right)^{m_{j}} T^{n_{j}} \mathcal{A}_{j} \exp \left(-\frac{\mathcal{E}_{j}}{\mathcal{R} T}\right)
$$

where $m_{j}, n_{j}, \mathcal{A}_{j}$, and $\mathcal{E}_{j}$ are model constants determined empirically by Thermal Gravimetric Analysis (TGA) . The rate of production of gases is the same as the rate of decomposition of the solid. Converting the mass fraction contributed by each reaction to mole density production, we get

$$
\pi_{i}=\epsilon_{m} \delta \rho_{p} \sum_{j=1}^{N_{p}}\left[\partial_{t} \xi_{j} F_{j} \widetilde{\gamma}_{j i}\right]
$$

where

$$
\widetilde{\gamma}_{j i}=\frac{\gamma_{j i}}{\sum_{k=1}^{N_{s}} \gamma_{j k} \mathcal{M}_{k}}
$$

\section{II.C. Mass conservation equation (Gas)}

The mass conservation for the gaseous phase is computed by multiplying the gaseous species conservation equation by $\mathcal{M}_{i}$ (molar mass of species $i$ ) and summing over $i$,

$$
\frac{\partial\left(\epsilon_{g} \rho_{g}\right)}{\partial t}+\epsilon_{g} \frac{\partial\left(\rho_{g} v_{g}\right)}{\partial x}=\pi_{\rho}
$$

\section{II.D. Momentum conservation equation}

The momentum equation in porous media was first derived empirically by Darcy (1856) using experimental correlations. Several versions, depending on the regime of interest, have been derived by various investigators (see Nield \& Bejan, ${ }^{6}$ 2006) using statistical concepts or the homogenization method. For the current study, we use Darcy's law in its original form to compute the average gas velocity,

$$
v_{g}=-\frac{K}{\mu} \frac{\partial p}{\partial x}
$$

where $K$ is Darcy's permeability coefficient, and $\mu$ is the dynamic viscosity.

\section{II.E. Energy conservation equation}

For PICA we estimate that the velocity of the pyrolysis gases inside the porous medium to be small and assume that the temperature of the pyrolysis gases will quickly equilibrate with the temperature of the solid phase. We assume that the three phases (fiber, matrix, and gas) are at the same temperature in a given averaging volume. The one-dimensional energy conservation equation is thereby approximated to read,

$$
\frac{\partial\left(\rho_{a} e_{a}\right)}{\partial t}+\frac{\partial\left(\rho_{g} h_{g} v_{g}\right)}{\partial x}=\frac{\partial}{\partial x}\left(k \frac{\partial T}{\partial x}\right)
$$

with $\rho_{a} e_{a}=\epsilon_{m} \rho_{m} h_{m}+\epsilon_{f} \rho_{f} h_{f}+\epsilon_{g} \rho_{g} e_{g}$.

The energy and the enthalpy of the gas phase, $e_{g}$ and $h_{g}$, are computed using the chemistry library MUTATION. The virgin and the char enthalpies, $h_{v}$ and $h_{c}$ are extracted from literature data.

\section{Numerical Method}

The strategy that we have followed for time integration of the governing equation is shown schematically in Fig. (1). We first advance the temperature one time step by assuming that the temperature distribution is mainly determined by the density, specific heat and conduction of the solid material, and that we can lag the gas density one time step. Having determined the temperature, the pyrolysis reaction is advanced one time step. This provides us with the gas production by pyrolysis. The gas density conservation equation is advanced with the pressure as the dependent variable. Having the density and the pressure, we determine the velocity and advance the molar density. 


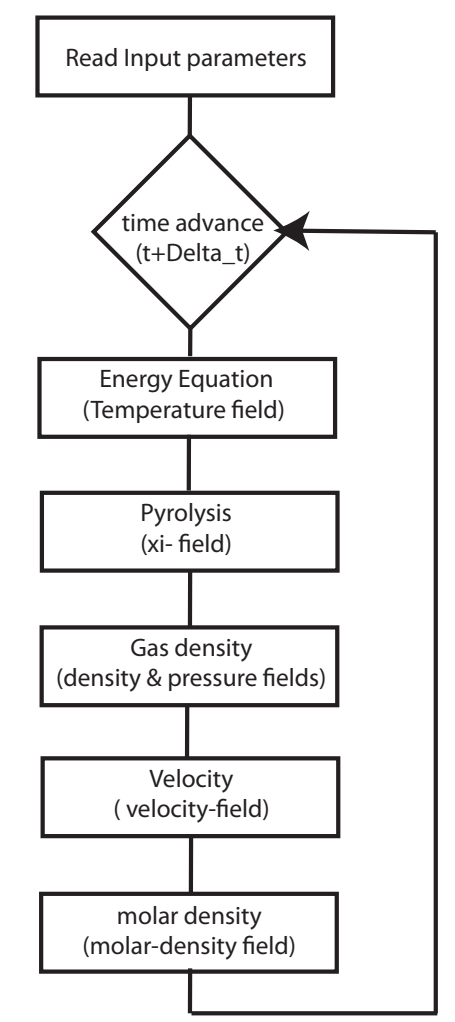

Figure 1. Time advancement strategy

We shall use the second order staggered mesh approximation as shown in Fig. 2 for spatial differencing where we place the velocity at the cell face and all other variables at the cell center. For integration in time, we shall follow a dual step approach where the equation are approximated using a second order implicit time differencing scheme combined with a Newton iteration.

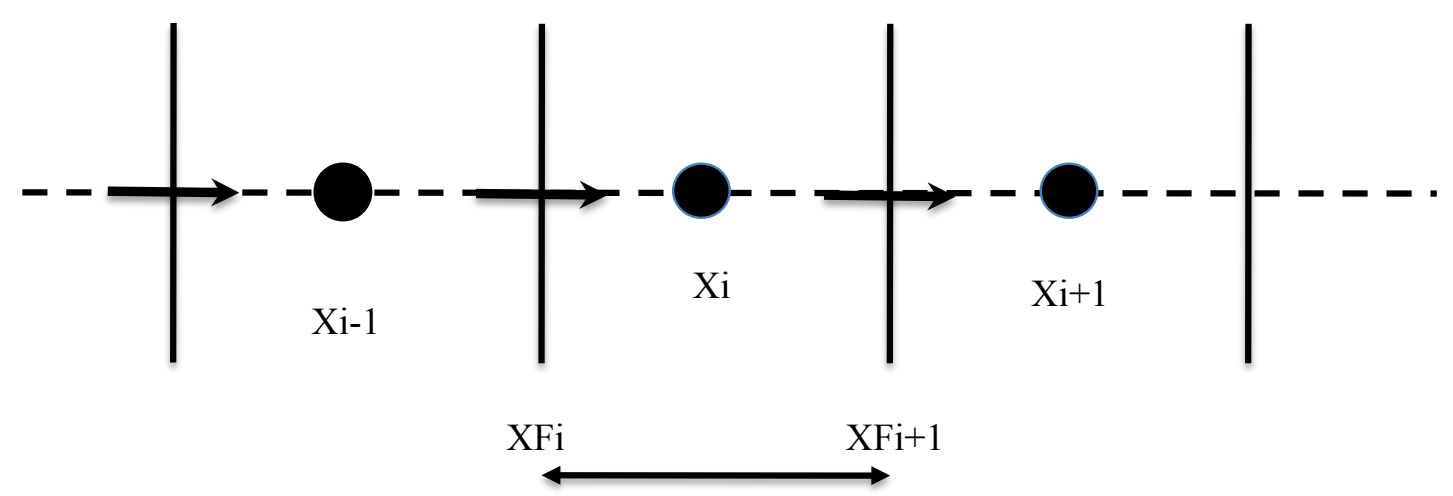

Figure 2. Staggered mesh arrangement where the velocity is defined at the faces, the variables are defined at the cell center

The conservation equations can be written in the general form,

$$
\frac{\partial q}{\partial t}+\frac{\partial F(q)}{\partial x}=S
$$

$$
4 \text { of } 10
$$


where $q$ is a dependent variable, and $S$ is a time dependent source term..

\section{III.A. Spatial discretization}

The conservation equation is discretized in space by multiplying Eq.9 by $d x$ and integrating over the grid interval,

$$
\int_{x_{i}^{F}}^{x_{i+1}^{F}} \frac{\partial q}{\partial t} d x+\left.F(q)\right|_{x_{i+1}^{F}}-\left.F(q)\right|_{x_{i}^{F}}=\int_{x_{i}^{F}}^{x_{i+1}^{F}} S d x
$$

where superscript, ${ }^{F}$, denotes face values. On a moving grid the above equations are recast in the form,

$$
\frac{\partial}{\partial t} \int_{x_{i}^{F}}^{x_{i+1}^{F}} q d x-\left(\left.q\right|_{x_{i+1}^{F}} \frac{\partial x_{i+1}^{F}}{\partial t}-\left.q\right|_{x_{i}^{F}} \frac{\partial x_{i}^{F}}{\partial t}\right)+\left.F(q)\right|_{x_{i+1}^{F}}-\left.F(q)\right|_{x_{i}^{F}}=\int_{x_{i}^{F}}^{x_{i+1}^{F}} S d x
$$

We then define the dependent variables to be cell-averaged variables,

$$
\int_{x_{i}^{F}}^{x_{i+1}^{F}} q d x=\langle q\rangle_{i} \Delta x_{i}^{F}
$$

where,

$$
\Delta x_{i}^{F}=x_{i+1}^{F}-x_{i}^{F}
$$

The discrete variables at the center of the cells, in this finite volume approximation, are volume averaged variables. Face values of cell-centered variables, $\left.q\right|_{x_{i}^{F}}$, are computed from the volume averaged variables to be,

$$
\left.q\right|_{x_{i}^{F}}=\frac{1}{2}\left(\langle q\rangle_{i}+\langle q\rangle_{i-1}\right)
$$

In what follows, we shall drop the brackets, \langle\rangle , for simplicity of notation.

\section{III.B. Second order dual time step integration in time}

We shall use a second order implicit scheme to discretize Eq.(11) in time,

$$
\begin{aligned}
\frac{1}{\Delta t}\left(\frac{3}{2} q_{i}^{n+1}\left(\Delta x_{i}^{F}\right)^{n+1}-2 q_{i}^{n}\left(\Delta x_{i}^{F}\right)^{n}+\frac{1}{2} q_{i}^{n-1}\left(\Delta x_{i}^{F}\right)^{n-1}\right) & \\
-\left.q\right|_{x_{i+1}^{F}} ^{n+1}\left(\frac{\delta x_{i+1}^{F}}{\delta t}\right)+\left.q\right|_{x_{i}^{F}} ^{n+1}\left(\frac{\delta x_{i}^{F}}{\delta t}\right) & \\
+\left.F\left(q^{n+1}\right)\right|_{x_{i+1}^{F}}-\left.F\left(q^{n+1}\right)\right|_{x_{i}^{F}} & =\int_{x_{i}^{F}}^{x_{i+1}^{F}} S^{n} d x
\end{aligned}
$$

where,

$$
\frac{\delta f}{\delta t}=\frac{1}{\Delta t}\left(\frac{3}{2} f^{n+1}-2 f^{n}+\frac{1}{2} f^{n-1}\right)
$$

is a second-order differentiation in time.

Newton iteration is used to find a solution to the nonlinear equation in $q^{n+1}$ using,

$$
\begin{aligned}
\left(\frac{3\left(\Delta x_{i}^{F}\right)^{n+1}}{2 \Delta t} \Delta q_{i}\right. & \left.+\left.\left(\left.\frac{\partial F}{\partial q}\right|^{k}-\frac{\delta x_{i+1}^{F}}{\delta t}\right) \Delta q\right|_{x_{i+1}^{F}}-\left.\left(\left.\frac{\partial F}{\partial q}\right|^{k}-\frac{\delta x_{i}^{F}}{\delta t}\right) \Delta q\right|_{x_{i-1}^{F}}\right) \\
= & -\frac{\left(\Delta x_{i}^{F}\right)^{n+1}}{\Delta t}\left[\frac{3}{2} q_{i}^{k}-2 q_{i}^{n} \frac{\left(\Delta x_{i}^{F}\right)^{n}}{\left(\Delta x_{i}^{F}\right)^{n+1}}+\frac{1}{2} q_{i}^{n-1} \frac{\left(\Delta x_{i}^{F}\right)^{n-1}}{\left(\Delta x_{i}^{F}\right)^{n+1}}\right] \\
& -\left(\left.F\left(q^{k}\right)\right|_{x_{i+1}^{F}}-\left.q\right|_{x_{i+1}^{F}} ^{k} \frac{\delta x_{i+1}^{F}}{\delta t}\right)+\left(\left.F\left(q^{k}\right)\right|_{x_{i}^{F}}-\left.q\right|_{x_{i}^{F}} ^{k} \frac{\delta x_{i}^{F}}{\delta t}\right)
\end{aligned}
$$




$$
+\int_{x_{i}^{F}}^{x_{i+1}^{F}} S^{n} d x
$$

where,

$$
\Delta q_{i}=\left(q_{i}^{k+1}-q_{i}^{k}\right)
$$

At convergence of the right hand side, we set $q_{i}^{n+1}=q_{i}^{k}$.

\section{Results}

A series of verification procedures have been used to test every routine in the code. In the paper we will expand on the section concerning verification of the code. For illustration purposes we quickly go over some of the features of the formulation in this extended abstract.

\section{IV.A. Thermal Conduction}

First and foremost, a material response code is a thermal conduction analysis code. The quantity of interest is the bond-line temperature, and the problem reduces to a surface heat-flux boundary condition combines with heat conduction to the bond-line. We set the gaseous properties to be zero, meaning $e_{g}, h_{g}, \rho_{g}, v_{g}$, and $X_{i}=0$. Only the solid properties remain active in the energy equation. By setting these properties to be constants, independent of space and temperature, analytical solutions exist to the heat conduction equation for both Dirichlet and Neuman boundary conditions.

\section{Neumann boundary conditions}

We compare our numerical solution to the time dependent conduction equation with the following conditions:

Initial condition:

$T=T_{0}$ at $t=0$, and

Boundary conditions:

For $t>0: \frac{\partial T}{\partial x}=0$ at $x^{\prime}=0, \frac{\partial T}{\partial x}=-\frac{\dot{q}}{k}$ at $x^{\prime}=L$ where $x^{\prime}=x-x_{0}$ and $L=x_{w}-x_{0}$.

An analytical solution exists for this problem in terms of an infinite series,

$$
T=\frac{\dot{q}}{2 k L}\left(\frac{L^{2}}{3}+4 L^{2} \sum_{n=1}^{\infty} \frac{(-1)^{n}}{(n \pi)^{2}} \exp \left(-\lambda_{n}^{2} \sigma^{2} t\right) \cos \left(\lambda_{n} x^{\prime}\right)-\left(x^{\prime 2}+2 \sigma^{2} t\right)\right)+T_{0}
$$

with $\lambda_{n}=\frac{n \pi}{L}$.

Figure 3 shows that the numerical and analytical solutions match perfectly. In addition to the implementation of the spatial differentiation and time integration, this test also verifies the implementation of the the boundary conditions. Many more verification cases will be presented in the paper.

\section{IV.B. Pyrolysis Gas compositions}

A main new feature of CAT is that it enables tracking the composition of the pyrolysis gases from their generation through the char region. We do not have detailed chemistry data for PICA, but we are in the process of developing a data base for this important material. Sykes studied the decomposition products of a phenolic formaldehyde resin in $1967 .^{7}$ It is the only complete quantitative study that we found so far on this type of resin. The $\mathrm{PhD}$ thesis of April, ${ }^{8}$ which is on a Nylon-Phenolic resin, shows very similar results (p. 138) that tend to confirm the data published by Sykes.

We have modeled a $2.5 \mathrm{~cm}$ slab of material with PICA properties using 4 global irreversible reactions plus carbon (the assumed composition of the charred matrix). The set of generated species and assumed reactions is shown in Table 1. 


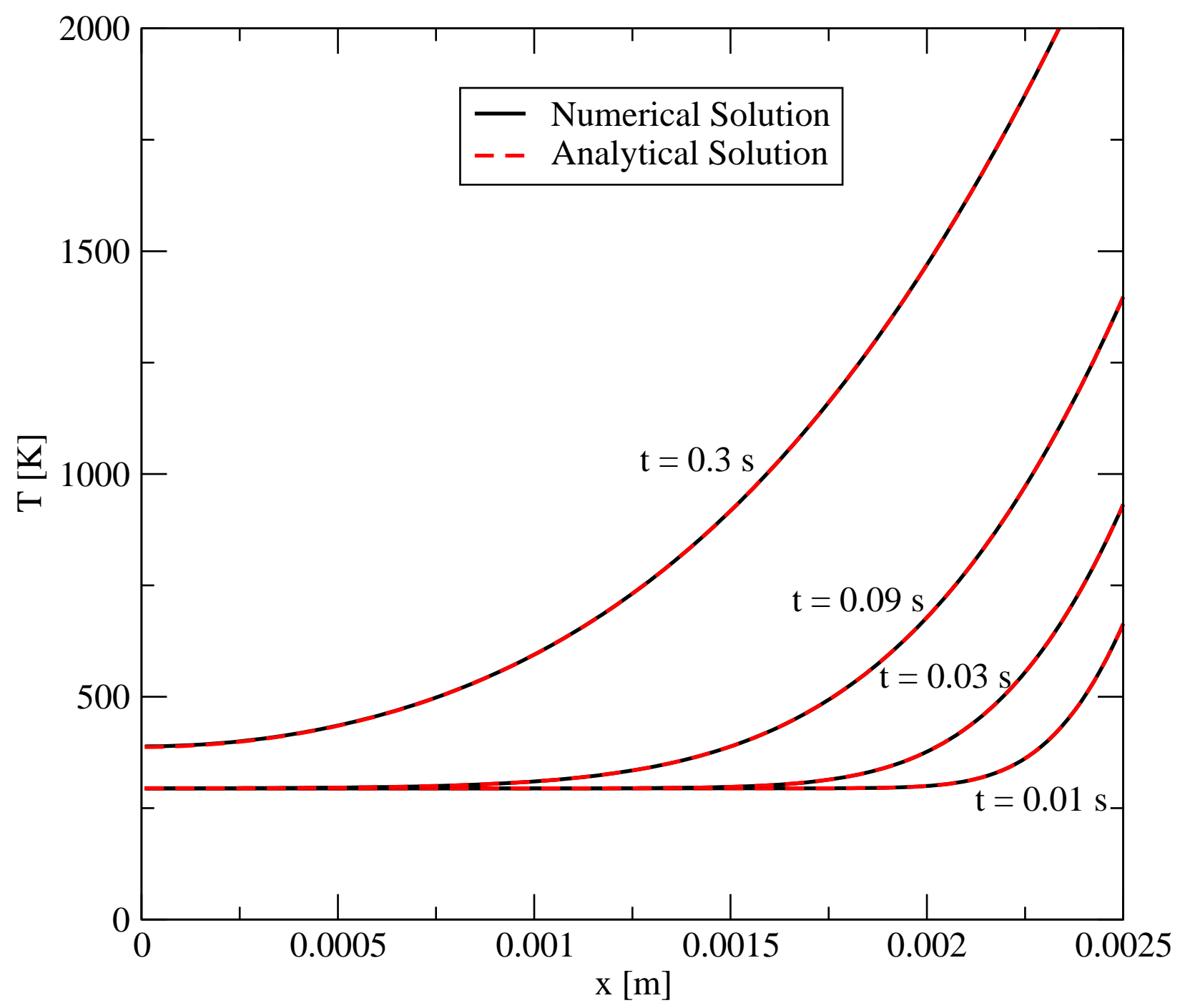

Figure 3. Staggered mesh arrangement where the velocity is defined at the faces, the variables are defined at the cell center

To illustrate the capability, we assume frozen chemistry within the materials, but compute the recession using equilibrium chemistry assumption at the surface. After $30 \mathrm{~s}$ of exposure time at $300 \mathrm{~W} / \mathrm{cm}^{2}$, the material has recessed about $7.5 \mathrm{~mm}$. The distribution of the species within the material is shown in Fig. 4 . We can recognize the two decomposition reactions, one producing mainly water and phenol, the other producing hydrogen, methane and carbon monoxide. More complete analysis of the results showing the velocity of the gas, the density distribution, and other quantities, as well as comparison with cases assuming equilibrium chemistry within the material will be discussed in the full paper.

\section{IV.C. Comparison with FIAT}

After Kendall et al. ${ }^{4}$ published their reference work that led to the CMA code, the pyrolysis species have been treated as being in equilibrium (this model is still in use in FIAT). In order to compare our results to FIAT, we use the same two decomposition reactions, each producing the same elemental gas composition as in FIAT. Figure 5 shows comparison between FIAT and CAT of the temperature evolution at various thermocouple locations. While the figure show poor agreement between the two codes for the lower thermocouples (at the bond-line and the first thermocouple), the major source of the discrepancy is due to the way the two 


\begin{tabular}{|c|c|c|c|c|c|c|c|}
\hline $\mathrm{j}$ & Pyrolysis balance equations & Peak & $F_{j}$ & $\mathcal{A}_{j}$ & $\mathcal{E}_{j}$ & $m_{j}$ & $n_{j}$ \\
\hline \hline & Model $($ Sykes + CMA/FIAT) & Sykes & Sykes & CMA & CMA & CMA & CMA \\
\hline 1 & $P F R_{1} \rightarrow \mathrm{H}_{2} O($ physisorbed $)$ & $100 C$ & 0.01 & $8.56 \cdot 10^{3}$ & $7.12 \cdot 10^{4}$ & 3 & 0 \\
2 & $P F R_{2} \rightarrow 0.69 \mathrm{H}_{2} \mathrm{O}+0.02 \mathrm{C}_{6} \mathrm{H}_{6}$ & $500 \mathrm{C}$ & 0.24 & $8.56 \cdot 10^{3}$ & $7.12 \cdot 10^{4}$ & 3 & 0 \\
& $+0.29 \mathrm{C}_{6} \mathrm{H}_{6} \mathrm{O}$ & & & & & & \\
3 & $P F R_{3} \rightarrow 0.09 \mathrm{CO}_{2}+0.33 \mathrm{CO}+0.58 \mathrm{CH}_{4}$ & $600 \mathrm{C}$ & 0.03 & $4.98 \cdot 10^{8}$ & $1.70 \cdot 10^{5}$ & 3 & 0 \\
4 & $P F R_{4} \rightarrow \mathrm{H}_{2}$ & $800 \mathrm{C}$ & 0.06 & $4.98 \cdot 10^{8}$ & $1.70 \cdot 10^{5}$ & 3 & 0 \\
5 & $P F R_{5} \rightarrow C$ & -- & 0.66 & 0 & 0 & 3 & 0 \\
\hline
\end{tabular}

Table 1. Pyrolysis balance equations and kinetic parameters.

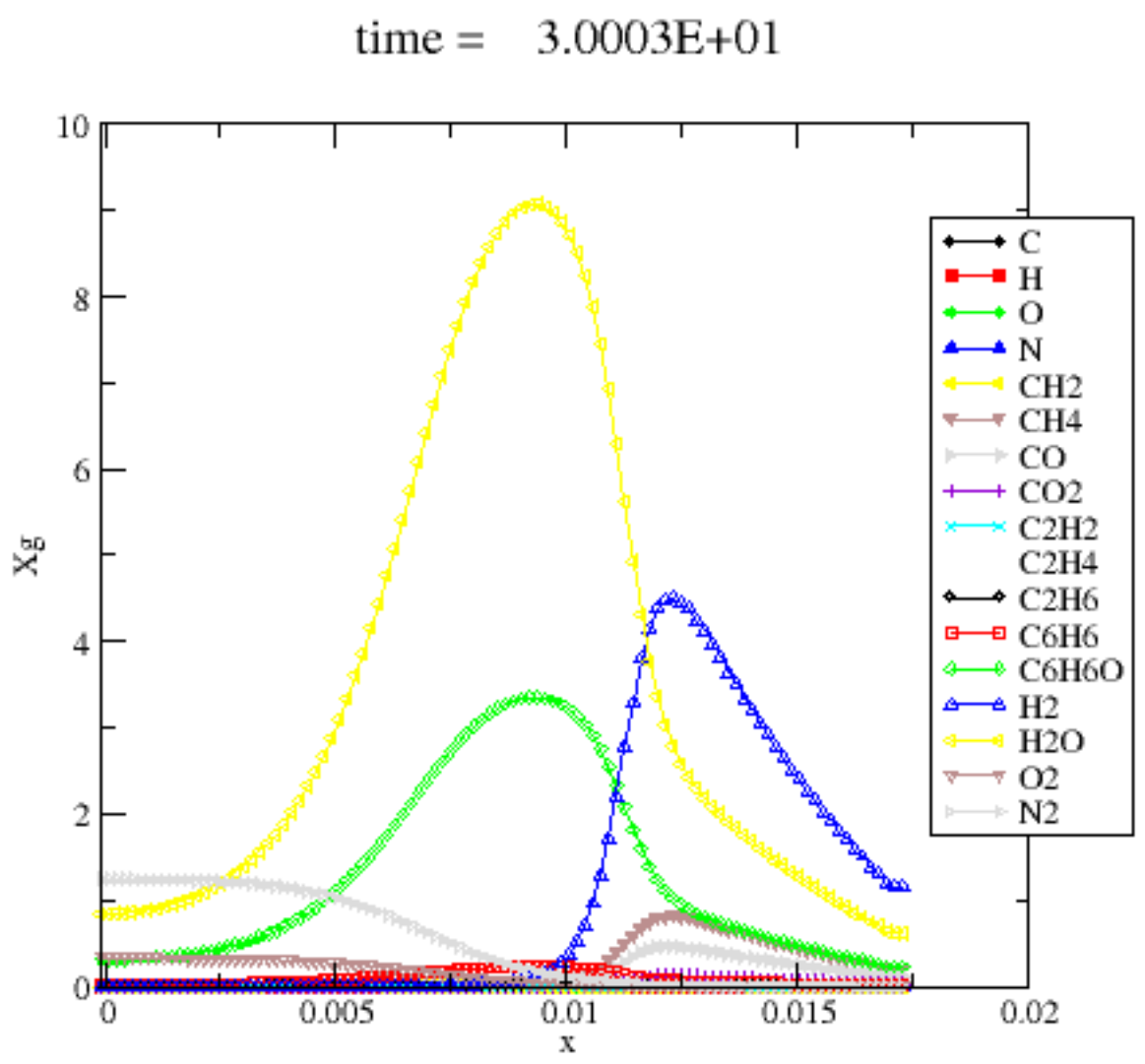

Figure 4. Mole density distribution for frozen chemistry at $t=30 \mathrm{~s}$.

codes compute the consumption of carbon $\left(\dot{m}_{c}\right)$. We point out that FIAT uses tables to compute $\dot{m}_{c}$, CAT computes the surface equilibrium chemistry at every time step. Figure 6 shows that while the difference between the two codes is small for both the pyrolysis gas production rate $\left(\dot{m}_{g}\right)$ and the char consumption rate $\left(\dot{m}_{c}\right)$, the error accumulates in time. For the case considered, FIAT estimates the total recession to be $8.24 \mathrm{~mm}$ for the $30 \mathrm{sec}$ exposure time, while CAT estimates the total recession to be $7.56 \mathrm{~mm}$, i.e. we have a $9 \%$ difference in the location of the hot surface after $30 \mathrm{sec}$. The problem is highly non-linear since the location of the surface where the boundary condition is imposed highly impacts the evolution of the temperature at a fixed location. 


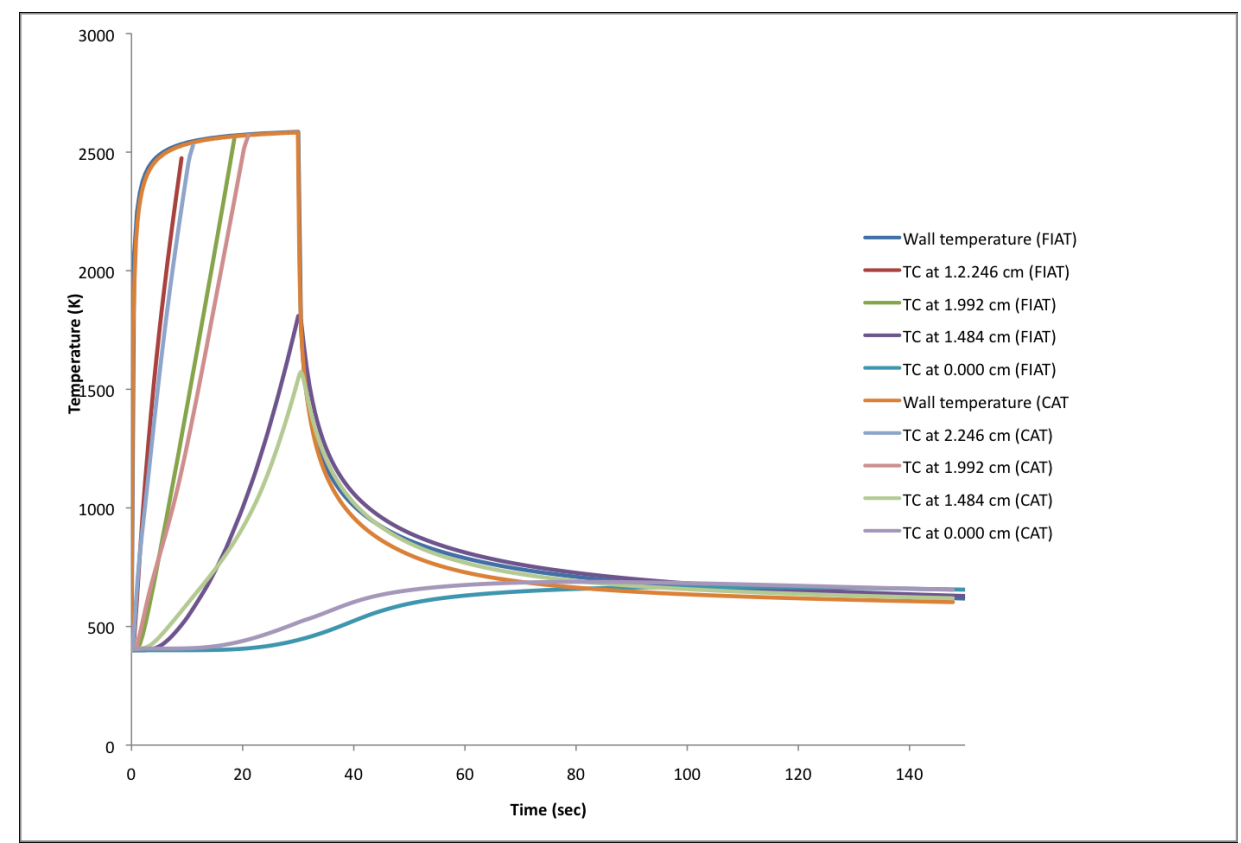

Figure 5. Comparison between FIAT and CAT of temperature evolution at various TC locations.

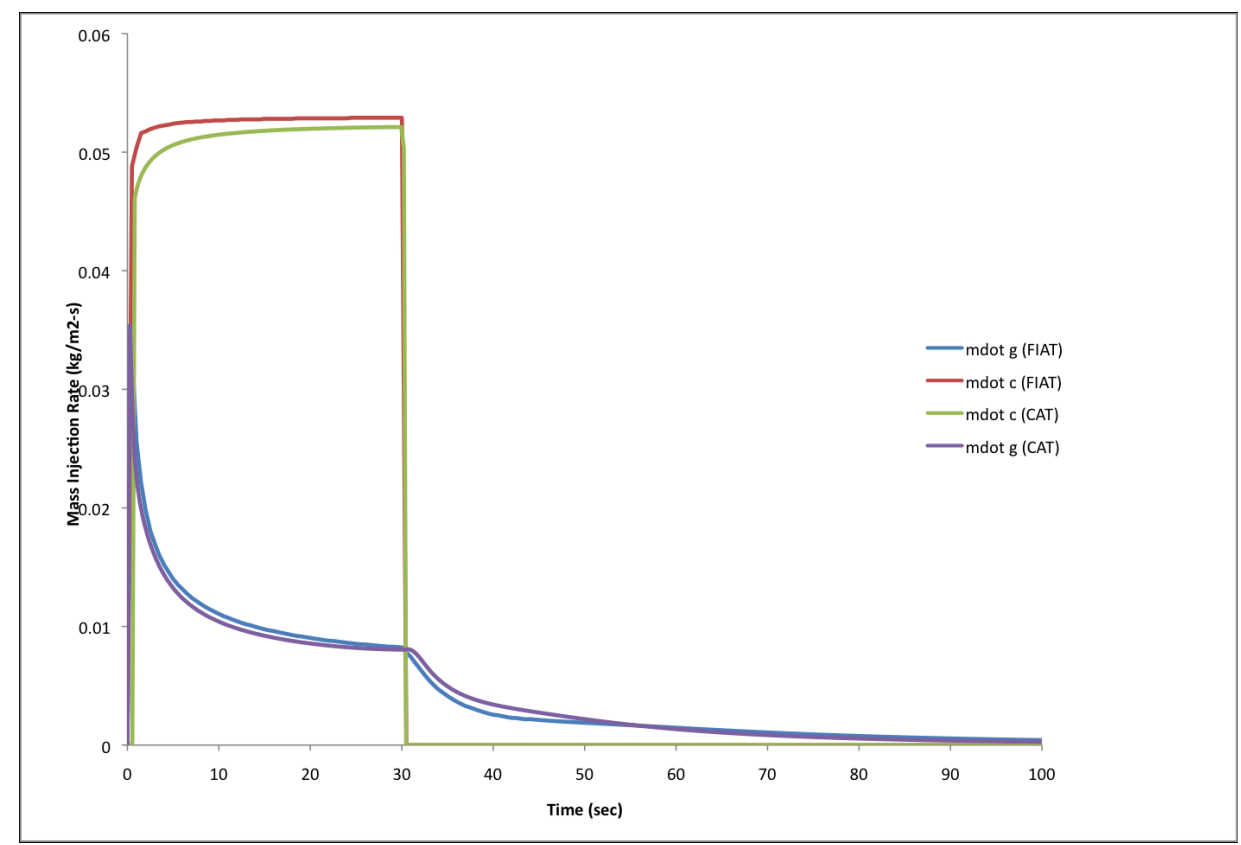

Figure 6. Comparison between FIAT and CAT of the computed gas mass flux and char consumption rate.

\section{Summary}

In the full paper a new material response code will be described that enables the simulation of the pyrolysis gas flow and chemistry within the material. The verification steps taken to test the code will be

fully described. This includes comparison of results with FIAT, the standard code used in the design of 
many of NASA's Thermal Protection Systems. Cases studying the sensitivity of the material response to the chemistry within the material will be described.

\section{Acknowledgments}

This research was supported by the fundamental aeronautics program - hypersonics (FAH) project. The interest and support of Dr. A. Calomino (Associate Project Investigator, Material and Structures) is gratefully acknowledge.

\section{References}

${ }^{1}$ Tran, H. K., Johnson, C. E., Rasky, D. J., Hui, F. C. L., Hsu, M.-T., Chen, T., Chen, Y. K., Paragas, D., and Kobayashi, L., "Phenolic Impregnated Carbon Ablators (PICA) as Thermal Protection Systems for Discovery Missions," NASA Technical Memorandum 110440, 1997.

${ }^{2}$ Stackpoole, M., Sepka, S., Cozmuta, I., and Kontinos, D., "Post-Flight Evaluation of Stardust Sample Return Capsule Forebody Heatshield Material," AIAA paper 2008-1202, Jan. 2008.

${ }^{3}$ Ritter, H., Portela, P., Keller, K., Bouilly, J. M., and Burnage, S., "Development of a European Ablative Material for Heatshields of Sample Return Missions," 6th European Workshop on TPS and Hot structures, Stuttgart, Germany, 1-3 April 2009.

${ }^{4}$ Kendall, R. M., Bartlett, E. P., Rindal, R. A., and Moyer, C. B., "An Analysis of the Coupled Chemically Reacting Boundary Layer and Charring Ablator: Part I," NASA CR 1060, 1968.

${ }^{5}$ Gnoffo P. A., Johnston C. O., and Thompson, R. A., "Implementation of Radiation, Ablation, and Free Energy Minimization Modules for Coupled Simulations of Hypersonic Flow," AIAA 2009-1399, 2009.

${ }^{6}$ Nield, D. A., and Bejan, A., "Convection in Porous Media", 3rd Edition, Springer, 2006.

${ }^{7}$ Sykes, G. F., "Decomposition characteristics of a char-forming phenolic polymer used for ablative composites," NASA TN D-3810, 1967, 20 p.

${ }^{8}$ April G. C., "Energy transfer in the char zone of a charring ablator," Louisiana State University, PhD thesis,1969, Also, NASA CR 107533. 